\title{
THE DEFLECTION OF A RECTANGULAR PLATE FIXED AT THE EDGES*
}

BY

H. W. MARCH

The problem of finding the deflection of a loaded rectangular plate fixed at the edges has offered considerable analytical difficulty. A very ingenious method of approximation capable of application to many other boundary value problems was applied to this problem by Ritzt who stated that the usual method of combining particular solutions of the appropriate differential equation is known to fail. Presumably his reason for this statement was that the latter method leads to an infinite system of equations in infinitely many variables whose solution presents difficulty. In the present treatment the method of combining particular solutions is used and the unique solution of the resulting infinite system of equations for the determination of the coefficients is given. The deflection is expressed in a form that appears to be simpler and better adapted to the discussion of the elastic surface than that given by Ritz. In the case of a uniformly loaded plate to which the discussion of this paper is confined, the deflection is found to a high degree of approximation by retaining the first term of the infinite series in the expression of the solution.

A treatment of the same subject, making use of particular solutions of the type used in this paper, was given, as I have recently discovered, by H. Hencky in a Darmstadt dissertation in 1913. He failed, however, to give an adequate discussion of the infinite system of equations to which he was led. In the present paper a fortunate choice of coefficients in the infinite series representing the deflection results in a much more tractable system of equations than Hencky's, the unknown coefficients decreasing much more rapidly in numerical value with increasing index. There results a very decided shortening of the numerical work. For example, Hencky finds for the first few coefficients in the case of a square plate

1.4138, $0.0945,-0.1093, \quad 0.0770, \quad-0.0510, \quad 0.0376$

The corresponding coefficients in this paper are

$0.167,-0.0010,-0.00044,-0.00015,-0.000026,-0.000015$.

* Presented to the Society, December 29, 1923.

$\dagger$ W. Ritz, Journal für Mathematik, vol. 135 (1908), pp.1-61. Also GesammelteWerke. 
Hencky's work has apparently escaped the attention of those interested in the theoretical aspects of elasticity.*

The discussion will be confined to the uniformly loaded plate. The method employed can probably be readily extended to the case of a load symmetrically distributed with respect to the center of the rectangle and probably without great difficulty to the case of an arbitrary distribution of load.

The solution in series. The deflection $\Psi$ of a uniformly loaded plate satisfies the differential equationt

$$
\nabla^{4} \Psi=A
$$

where

$$
A=\frac{p}{\frac{2}{3} E h^{2} /\left(1-\sigma^{2}\right)}
$$

$p$ being the uniform load, $h$ one-half the thickness of the plate, $E$ and $\sigma$ elastic constants of the material of the plate.

For a rectangular plate clamped at the edges $\Psi$ is subject to the boundary conditions

$(2$, a) $\quad \boldsymbol{\Psi}=0, \quad x=0, \quad x=a, \quad y=0, \quad y=b$

$(2, \mathrm{~b}) \quad \frac{\partial \Psi}{\partial x}=0, \quad x=0, \quad x=a, \quad \frac{\partial \Psi}{\partial y}=0, \quad y=0, \quad y=b$.

Under these conditions (1) has not more than one $\S$ solution. To obtain the solution, let

$$
\boldsymbol{H}_{1}=a x(x-a) y(y-b),
$$

where

$$
\varkappa=\frac{A}{8} \text {. }
$$

$\Psi_{1}$ satisfies the differential equation (1) and the boundary conditions $(2, \mathrm{a})$ but does not satisfy the conditions $(2, b)$, since

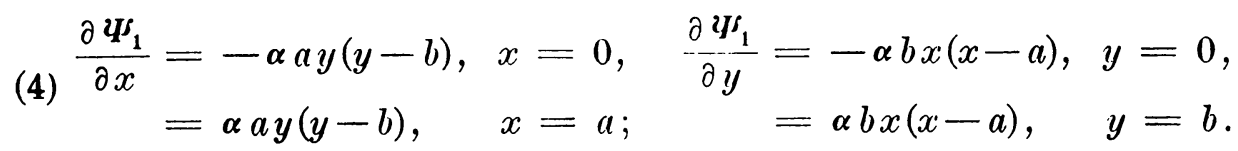

To satisfy the remaining boundary conditions $(2, b)$ add to $\Psi_{1}$ a properly chosen solution of

$$
\nabla^{4} \Psi s=0
$$

* Love, Theory of Elasticity, 3d edition, 1920, p. 496.

† Love, p. 489.

$\ddagger$ Love, p. 470 and p. 489.

§. Mathieu, Journal de Mathématiques, vol. 14 (1869), p. 392. 
If $\xi(x, y)$ is a solution of $\nabla^{2} \xi=0$ it is readily found that

$$
\Psi=\imath(x, y) \xi(x, y)
$$

is a solution of (5) if $u$ is of the form

$$
u=h\left(x^{2}+y^{2}\right)+k x+l y+m .
$$

Choose the following solution of (5) which is built up from solutions of the type (6):

where

$$
\begin{aligned}
\Psi_{2}= & \alpha\left\{\sum_{n} \frac{a_{n}}{\lambda_{n} b+\sinh \lambda_{n} b}\left[(y-b) \sinh \lambda_{n} y+y \sinh \lambda_{n}(y-b)\right] \sin \lambda_{n} x\right. \\
& \left.+\sum_{m} \frac{b_{m}}{\sigma_{m} a+\sinh \sigma_{m} a}\left[(x-a) \sinh \sigma_{m} x+x \sinh \sigma_{m}(x-a)\right] \sin \sigma_{m} y\right\}
\end{aligned}
$$

$$
\lambda_{n}=\frac{n \pi}{a}, \quad \sigma_{m}=\frac{m \pi}{b} .
$$

Since the solution $\Psi_{1}$ and the boundary conditions $(2, \mathrm{~b})$ are symmetrical with respect to the center of the rectangle, it is evident that only odd indices $n$ and $m$ will occur in the series in (7). Hereafter $\Sigma_{m}$ and $\Sigma_{n}$ will be understood to refer to summations taken over the odd integers from 1 to $\infty$ unless otherwise noted.

Write

$$
\Psi \boldsymbol{\Psi}=\Psi \boldsymbol{s}_{1}+\Psi \boldsymbol{s}_{2},
$$

and note that $\Psi$ satisfies the conditions (2,a). The coefficients $a_{n}$ and $b_{m}$ are to be so determined that the conditions $(2, b)$ are satisfied:

$$
\begin{aligned}
\left(\frac{\partial \Psi}{\partial x}\right)_{x=0}= & \iota\{-a y(y-b) \\
& +\sum_{n} \frac{\lambda_{n} a_{n}}{\lambda_{n} b+\sinh \lambda_{n} b}\left[(y-b) \sinh \lambda_{n} y+y \sinh \lambda_{n}(y-b)\right] \\
& \left.-\sum_{m} b_{m} \sin \sigma_{m} y\right\}=0,
\end{aligned}
$$

$$
\begin{aligned}
& \left(\frac{\partial \Psi s}{\partial y}\right)_{y-0}=\alpha\left\{-b x(x-a)-\sum_{n} a_{n} \sin \lambda_{n} x\right. \\
& \left.\quad+\sum_{m} \frac{\sigma_{m} b_{m}}{\sigma_{m} a+\sinh \sigma_{m} a}\left[(x-a) \sinh \sigma_{m} x+x \sinh \sigma_{m}(x-a)\right]\right\}=0 .
\end{aligned}
$$

If these conditions are satisfied, it is evident from the symmetry with respect to the center of the rectangle that the conditions on the edges $x=a$ and $y=b$ are also satisfied. 
Equation (10) is satisfied if

$$
b_{m}=\frac{2}{b} \int_{0}^{b}\{-a y(y-b)
$$

$\left.+\sum_{n} \frac{\lambda_{n} a_{n}}{\lambda_{n} b+\sinh \lambda_{n} b}\left[(y-b) \sinh \lambda_{n n} y+y \sinh \lambda_{n}(y-b)\right] \sin \sigma_{m} y\right\} d y$.

The inversion of the order of summation with respect to $m$ and $n$ that is here implied is seen to be justified by the values found for $a_{n}$ and $b_{m}$. For it is found that $\left|a_{n}\right|<H /\left(\lambda_{n}^{5 / 2}\right), H$ a fixed positive number, and $b_{m}$ satisfies a similar condition. If the second term in (10) be written $\sum_{n} \Sigma_{m} A_{n, m} \sin \sigma_{m} y$ the coefficients $A_{n, m}$ then satisfy the condition

$$
\left|A_{n, m}\right|<\frac{D \sigma_{m}}{\left(\hat{\lambda}_{n}^{2}+\sigma_{m}^{2}\right)^{2}}, \quad D \text { a fixed positive number. }
$$

Hence the order of summation can be interchanged.

It follows readily from $(10, a)$ that

$$
b_{m}=\frac{8 a}{b \sigma_{m}^{3}}-\frac{8 \sigma_{m}}{b} \sum_{n} \frac{\lambda_{n}^{2}}{\left(\dot{\lambda}_{n}^{2}+\sigma_{m}^{2}\right)^{2}} \varrho\left(\lambda_{n} b\right) a_{n},
$$

where

$$
\varrho\left(\lambda_{n} b\right)=\frac{1+\cosh \lambda_{n} b}{\lambda_{n} b+\sinh \lambda_{n} b}
$$

In like manner from (11)

Writing:

$$
a_{n}=\frac{8 b}{a \lambda_{n}^{3}}-\frac{8 \lambda_{n}}{a} \sum_{m} \frac{\sigma_{m}^{2}}{\left(\lambda_{n}^{2}+\sigma_{m}^{2}\right)^{2}} \varrho\left(\sigma_{m} a\right) b_{m} .
$$

$$
b=\eta a
$$

where $\eta$ is taken to be less than or equal to 1 and interchanging $m$ and $n$ in (12), (12) and (14) become

$$
\begin{aligned}
& b_{n}=\frac{8 \eta^{2} a^{3}}{n^{3} \pi^{3}}-\frac{8 n \eta^{2}}{\pi} \sum_{m} \frac{m^{2}}{\left(m^{2} \eta^{2}+n^{2}\right)^{2}} \varrho(m \pi \eta) a_{m} \\
& a_{n}=\frac{8 \eta a^{3}}{n^{3} \pi^{3}}-\frac{8 n \eta^{2}}{\pi} \sum_{m} \frac{m^{2}}{\left(m^{2}+n^{2} \eta^{2}\right)^{2}} \varrho\left(\frac{m \pi}{\eta}\right) b_{m}
\end{aligned}
$$

The function $\rho$ occurring in these equations does not differ much from unity for most values of its argument and its presence does not materially complicate the numerical work of solving these equations. 
The square plate. The simplest case is that of the square $b=a$, i. e., $\eta=1$. In this case $a_{n}=b_{n}$ and (16) and (17) become identical, viz.,

$$
b_{n}=\frac{8 a^{3}}{n^{3} \pi^{3}}-\frac{8 n}{\pi} \sum_{m} \frac{m^{2}}{\left(m^{2}+n^{2}\right)^{2}} \varrho(m \pi) b_{m},
$$

or

$$
b_{n}=\frac{K}{n^{3}}-\sum_{m} \alpha_{n m} b_{m} \quad(n=1,3,5, \cdots),
$$

where

$$
K=\frac{8 a^{3}}{\pi^{3}}, \quad \alpha_{n m}=\frac{8}{\pi} \frac{n m^{2}}{\left(n^{2}+m^{2}\right)^{2}} \varrho(m \pi) .
$$

Equations (19) can be written

(21) $\alpha_{n 1} b_{1}+\alpha_{n 3} b_{3}+\cdots+\left(1+\alpha_{n n}\right) b_{n}+\cdots=\frac{K}{n^{3}} \quad(n=1,3,5, \cdots)$.

The coefficient of the term in the principal diagonal rapidly approaches 1 as a limit. Before discussing the solution of the infinite system of equations (21) the first four equations of the system will be written down:

$$
\begin{aligned}
& 1.546 b_{1}+0.229 b_{3}+0.094 b_{5}+0.050 b_{7}+\cdots=K, \\
& 0.065 b_{1}+1.212 b_{3}+0.165 b_{5}+0.111 b_{7}+\cdots=0.03705 K, \\
& 0.016 b_{1}+0.099 b_{3}+1.128 b_{5}+0.114 b_{7}+\cdots=0.008 K, \\
& 0.0061 b_{1}+0.048 b_{3}+0.081 b_{5}+1.091 b_{7}+\cdots=0.00292 K .
\end{aligned}
$$

It will now be shown by a slight modification of an argument due to von Koch* that the system (21) has one and only one solution. For the sake of easy reference to von Koch's paper, replace the unknowns $b_{1}, b_{3}, \ldots$ by $x_{1}, x_{3}$ etc., and write (21) as

$$
\alpha_{i 1} x_{1}+\alpha_{i 3} x_{3}+\cdots+\left(1+\alpha_{i i}\right) x_{i}+\cdots=\frac{K}{i^{3}} \quad(i=1,3,5, \ldots)
$$

where

$$
\alpha_{i k}=\frac{8}{\pi} \frac{i k^{2}}{\left(i^{2}+k^{2}\right)^{2}} \frac{1+\cosh k \pi}{k \pi+\sinh k \pi}<\frac{8}{\pi} \frac{i k^{2}}{\left(i^{2}+k^{2}\right)^{2}} .
$$

Divide (23) by $\left(1+\alpha_{i i}\right)$ and set

$$
\frac{\alpha_{i k}}{1+\alpha_{i i}}=-l_{i k}, \quad b_{i i}=0, \quad \frac{K}{i^{3}\left(1+\alpha_{i i}\right)}=c_{i} .
$$

${ }^{*}$ H. von Koch, Jahresbericht der Deutschen Mathematiker-Vereinigung, vol. 22 (1913), pp. 285-291. 
Then

Equations (23) become

$$
\left|b_{i k}\right|<\frac{8}{\pi} \frac{i k^{2}}{\left(i^{2}+k^{2}\right)^{2}}, \quad C_{i}<\frac{K}{i^{3}} .
$$

$$
x_{i}-\sum_{k} b_{i k} x_{k}=C_{i} \quad(i=1,3,5, \ldots) .
$$

Let

Substitute and obtain

$$
x_{k}=x_{k}^{(1)}+C_{k} \text {. }
$$

$$
x_{i}^{(1)}-\sum_{k} l_{i k:} x_{l_{i}}^{(1)}=\sum_{k} b_{i k} C_{k}^{v}=C_{i}^{(1)} .
$$

Let

$$
x_{i}^{(1)}=x_{k}^{(2)}+C_{k}^{(1)},
$$

and so on, continuing this process of successive approximation indefinitely.

Using the notation

$$
b_{i k}^{(2)}=\sum_{v} b_{i v} b_{v k}, \quad b_{i k}^{(3)}=\sum_{v} b_{i v}^{(2)} b_{v k}, \text { etc., }
$$

it will be shown that the series

i. e.

$$
x_{i}=C_{i}+\gamma_{i}^{(1)}+C_{i}^{(2)}+\cdots,
$$

$$
x_{i}=C_{i}+\sum_{k} b_{i k} C_{k}+\sum_{k} b_{i k}^{(2)} C_{k}+\sum_{k} b_{i k}^{(3)} C_{k}+\cdots
$$

is absolutely convergent and that it represents the unique solution of the system (24), subject to the condition $\left|x_{k}\right|<X$, a fixed number. Von Koch's argument assumes that $\sum_{k}\left|b_{i k}\right|<\varepsilon<1, \varepsilon$ the same for all values of $i$. This condition is not satisfied in this case, although it is true that $\sum_{k}\left|b_{i k}\right|<1$. Accordingly his argument has been modified, taking advantage of the fact that in the present case $C_{k}<K / k^{3}$ instead of being simply less than a fixed number. This modification results in showing the dependence of $x_{i}$ on $i$ which is important for the discussion of the derivation of the system of equations (12) and (14). We have

$$
\sum_{k}\left|b_{i k} C_{k}\right|<\frac{8}{\pi} K \sum_{k} \frac{i k^{2}}{\left(i^{2}+k^{2}\right)^{2}} \frac{1}{k^{3}}<\frac{8 K i}{\pi} \sum_{k} \frac{1}{\sqrt{k}\left(i^{2}+l^{2}\right)^{2}} .
$$

Noting that the summation is over odd integers and taking account of the situation at the lower limit, it is readily found that

$$
\begin{aligned}
\sum_{l k} \frac{1}{\sqrt{\bar{k}\left(i^{2}+k^{2}\right)^{2}}} & <\frac{1}{2} \int_{0}^{\infty} \frac{d k}{\sqrt{k}\left(i^{2}+k^{2}\right)^{2}} \\
& =\frac{1}{2 i^{7 / 2}} \frac{I\left(\frac{1}{4}\right) \Gamma\left(\frac{7}{4}\right)}{2 \Gamma(2)}=\frac{1}{i^{7 / 2}} \frac{3}{8 \sqrt{2}} .
\end{aligned}
$$


Hence

$$
\begin{aligned}
\sum_{k}\left|b_{i k} C_{k}\right| & <\frac{3}{\pi \sqrt{2}} \frac{K}{i^{5 / 2}} \\
\sum_{k}\left|b_{i k}^{(2)} C_{k}\right| & \leqq \sum_{k} \sum_{v}\left|b_{i v}\right|\left|b_{v k}\right| C_{k} \\
& <\frac{3 K}{\pi \sqrt{2}} \sum_{v} \frac{\left|b_{i v}\right|}{v^{5 / 2}}<\frac{3 K}{\pi \sqrt{2}} \frac{8 i}{\pi} \sum_{v} \frac{1}{\left(i^{2}+v^{2}\right)^{z} \sqrt{v}} \\
& <\left(\frac{3}{\pi \sqrt{2}}\right)^{2} \frac{K}{i^{5 / 2}} .
\end{aligned}
$$

In general

Hence

$$
\sum_{k}\left|b_{i k}^{(p)} C_{k}\right|<\left(\frac{3}{\pi \sqrt{2}}\right)^{p} \frac{K}{i^{5 / 2}}
$$

$$
\left|x_{i}\right|<\frac{K}{i^{52}}\left(1+\varepsilon+\varepsilon^{2}+\cdots\right)=\frac{K}{i^{5 / 2}} \frac{1}{1-\varepsilon},
$$

where

$$
\varepsilon=\frac{3}{\pi \sqrt{2}}=0.677
$$

Hence the absolute convergence of (25) is established. The proof that (25) is a solution of (24) now follows immediately, exactly as in von Koch's article. That it is the only solution subject to the restriction $\left|x_{k}\right|<X$, a fixed number, follows also from the method of von Koch, noting that with

$$
A_{i}=\frac{8 i}{\pi}, \quad \varepsilon=\frac{3}{\pi \sqrt{2}}, \quad \text { and } \quad H=\sum_{k} \frac{1}{k^{8 / 2}}
$$

it follows that

$$
\begin{gathered}
\sum_{k}\left|b_{i k}\right|<1, \quad\left|b_{i k}^{(2)}\right|<A_{i} \varepsilon \frac{1}{k^{3 / 2}}, \quad \sum_{k}\left|b_{i k}^{(2)}\right|<A_{i} \varepsilon H \\
\sum_{k}\left|b_{i k}^{(3)}\right|<A_{i} \varepsilon^{2} H \quad \text { and } \quad \sum_{k}\left|b_{i k}^{(p)}\right|<A_{i} \varepsilon^{p-1} H
\end{gathered}
$$

It is to be observed that these inequalities would have been sufficient to show the absolute convergence of (25) but would not have shown the important fact that $\left|x_{i}\right|<M / i^{5 / 2}$ where $M$ is a fixed number.

Solving the system (21) using the formula (25) it is found that

$$
b_{1}=0.6477 \mathrm{~K}, \quad b_{3}=-0.0040 \mathrm{~K} \text {. }
$$

These values are found more easily by solving the first equation for $b_{1}$, retaining only the first term, using this value in the second equation to 
obtain an approximate value of $b_{3}$ by retaining the first two terms only, then correcting the value of $b_{1}$ by substituting the value found for $b_{3}$ in the first equation and so on. By this method it is found that

$$
b_{1}=0.6475 K, \quad b_{3}=-0.0040 K,
$$

in agreement with the foregoing results. By the same method it is also found that $b_{5}=-0.0017 K, b_{7}=-0.006 K, \quad b_{9}=-0.0003 K$, $b_{11}=-0.0001 K, \quad b_{13}=-0.00006 K$. This method of successive approximation though lacking complete justification can be applied with greater confidence when it is known that a solution exists and when it is known that the unknowns $b_{i}$ decrease very rapidly in absolute value, being subject as shown to the relation

$$
b_{i}<\frac{M}{i^{5 / 2}}
$$

Consequently the infinite series constituting the left-hand members of (21) converge very rapidly.

The deflection at any point of a square plate is given by (9) where $\Psi_{1}$ and $\Psi_{2}$ are defined by (3) and (7) respectively. It is easy to see from the values just found for the coefficients that it is necessary to retain in $\Psi_{2}$ only the terms containing $a_{1}=b_{1}$.

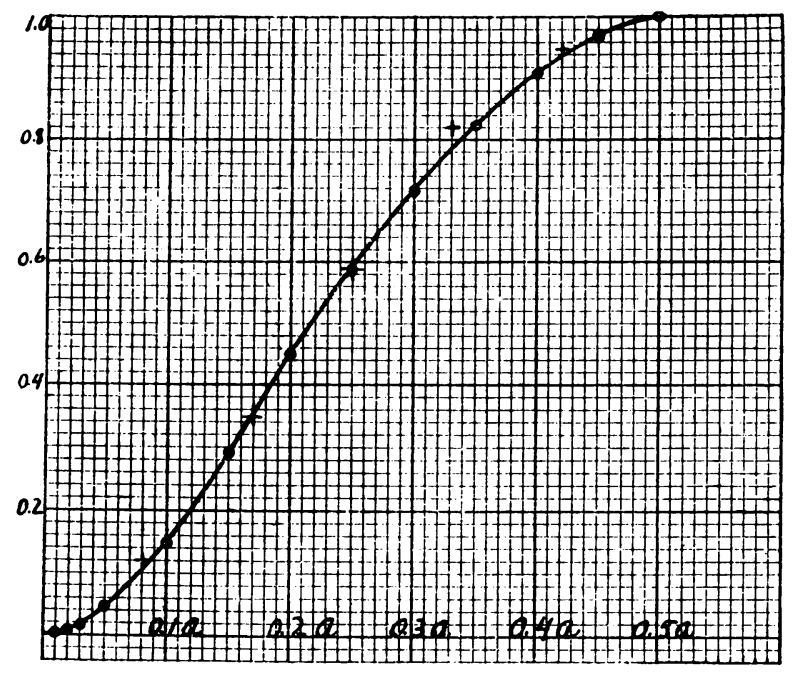

Deflection along a diameter of a square plate

In the accompanying figure, the deflection along a median line, $x==0.5 a$, of a square plate is shown in terms of the deflection at the center. The circles indicate points determined by the present formula. The crosses 
indicate results for one of the square plates experimentally investigated by Crawford.* The results for this and other plates were tabulated by Knott for comparison with the results of the application of Ritz's method.

The rectangular plate. In (16) and (17) replace $b_{m}$ by $x_{m+1}$ and $a_{m}$ by $x_{m}, m$ being odd in both cases, so that an even subscript correspond to a $b$, an odd subscript to an $a$. The equations (16) and (17) can then be written

$$
\begin{aligned}
x_{1}+a_{12} x_{2}+a_{14} x_{4}+a_{16} x_{6}+a_{18} x_{8}+\cdots & =K=C_{1}, \\
a_{21} x_{1}+x_{2}+a_{23} x_{3}+a_{25} x_{5}+a_{27} x_{7} & =K_{1}=C_{2}, \\
a_{32} x_{2}+x_{3}+a_{34} x_{4}+a_{36} x_{6}+a_{38} x_{8} & =\frac{K}{27}=C_{3}, \\
a_{41} x_{1}+a_{43} x_{3}+x_{4}+a_{45} x_{5}+a_{47} x_{7} & =: \frac{K_{1}}{27}=C_{4}, \\
a_{58} x_{2}+a_{54} x_{4}+x_{5}+a_{56} x_{6}+a_{58} x_{8} & =\frac{K}{125}=C_{5}, \\
a_{61} x_{1}+a_{83} x_{3}+a_{65} x_{5}+x_{6}+a_{67} x_{7} & =\frac{K}{125}=C_{6},
\end{aligned}
$$

where

$$
\begin{array}{rlrl}
a_{n m} & =\frac{8 \eta^{2}}{\pi} \frac{n(m-1)^{2}}{\left[n^{2} \eta^{2}+(m-1)^{2}\right]^{2}} \varrho\left(\frac{(m-1) \pi}{\eta}\right), & & n \text { odd, } m \text { even, } \\
& =\frac{8 \eta^{2}}{\pi} \frac{(n-1) m^{2}}{\left[(n-1)^{2}+m^{2} \eta^{2}\right]^{2}} \varrho(m \pi \eta), \quad n \text { even, } m \text { odd, } \\
& =0, \quad n \text { and } m \text { both even or both odd and } n \neq m, \\
& =1 \quad n=m,
\end{array}
$$

and

$$
K=\frac{8 \eta a^{3}}{\pi^{3}}, \quad K_{1}=\frac{8 \eta^{2} a^{3}}{\pi^{3}}
$$

and

$$
\begin{aligned}
C_{n} & =\frac{K}{n^{3}}, \quad n \text { odd } \\
& =\frac{K_{1}}{n^{3}}, \quad n \text { even }
\end{aligned}
$$

and

$$
K_{1}<K
$$

*W. T. Crawford, Proceedings of the Royal Society of Edinburgh, vol. 32 (1911-12), pp. 348--389.

† C. G. Knott, Proceedings of the Royal Society of Edinburgh, vol. 32 (1911-12), pp. 390-392. 
Adopting the notation used in the preceding section, let

$$
b_{i k}=-a_{i k}, \quad i \neq k \quad \text { and } \quad b_{i i}=0
$$

and use the method of successive approximation used in solving (24). In the following discussion $\sum_{k_{0}} \sum_{k_{0}}$ will indicate summation over even integers and odd integers respectively. When no subscript is written, the reader can readily determine which type of summation is meant. It is found that if $i$ is odd and $\eta$ so large that $\varrho(\pi \eta)<1$,

$$
\begin{aligned}
& \sum_{k_{e}}\left|b_{i k} C_{k}\right|<\frac{\eta^{z}}{\pi} K \sum_{k_{o}} \frac{i k^{2}}{\left(i^{2} \eta^{2}+k^{2}\right)^{2}} \frac{1}{k^{3}}<\frac{\varepsilon}{\eta^{3 / 2}} \frac{K}{i^{5 / 2}}, \\
& \sum_{k_{o}}\left|b_{i k}^{(2)} C_{k}\right|<\varepsilon^{2} \frac{K}{i^{5 / 2}},
\end{aligned}
$$

and in general

$$
\begin{aligned}
\sum_{k}\left|b_{i k}^{(p)} C_{k}\right| & <\varepsilon^{p} \frac{K}{i^{5 / 2}}, \quad p \text { even, } \\
& <\frac{\varepsilon^{p}}{\eta^{3 / 2}} \frac{K}{i^{5 / 2}}, \quad p \text { odd }
\end{aligned}
$$

Hence the series corresponding to (25) is absolutely convergent.

If $\eta$ is so small that $\varrho(\pi \eta)$ is greater than 1 , take out the factor $\varrho(\pi \eta)$ from each sum taken with respect to an odd second index. The result is that in case $i$ is odd the factor $\varrho(\pi \eta)$ will appear in the first and second inequalities, the factor $[\varrho(\pi \eta)]^{2}$ in the third and fourth, etc. Consequently

$$
\begin{aligned}
& \sum_{k}\left|b_{i k} C_{k}\right|+\sum_{k}\left|b_{i k}^{(2)} C_{k}\right|+\cdots \\
& \quad<\frac{K \varepsilon \varrho(\pi \eta)\left(1+\varepsilon \eta^{3 / 2}\right)}{i^{5 / 2} \eta^{3 / 2}}\left\{1+\varepsilon^{2} \varrho(\pi \eta)+\varepsilon^{4}[\varrho(\pi \eta)]^{2}+\cdots\right\} .
\end{aligned}
$$

This series is convergent if $\varepsilon^{2} \varrho(\pi \eta)<1$, i. e., if $\varrho(\pi \eta)<2.198$. This is the case if $\eta>0.15$. This means that convergence is assured for most cases of practical interest.

When $i$ is even the same methods apply. It is to be observed that the factor $i^{5 / 2}$ which appeared in the denominator in the foregoing inequalities is now replaced by $(i-1)^{5 / 2}$.

That (25) is a solution follows as in the case of the square. The proof of the uniqueness of the solution (25) requires that as in the case of the square

$$
\lim _{p=\infty} \sum_{k}\left|b_{i k}^{(p)}\right|=0
$$


This can readily be shown to be true if

$$
\varepsilon^{2} \varrho(\pi \eta)<1 \text {, i. e., if } \eta>0.15
$$

It has been shown that if $i$ is odd

$$
x_{i}=a_{i} \mid<\frac{M}{i^{5 / 2}}
$$

if $i$ is even

$$
\left|x_{i}\right|=\left|b_{i-1}\right|<\frac{M}{(i-1)^{5 / 2}}, \quad \text { i. e., } \quad\left|b_{m}\right|<\frac{M}{m^{5 / 2}} .
$$

The present method of obtaining solutions of the system (27) is not established for $\eta$ less than 0.15 , i. e., for extremely narrow rectangles. As in most cases of practical interest $\eta$ is greater than 0.15 it does not seem worth while to attempt to modify the proof to include the case of these small values of $\eta$.

Again it appears from the rapid decrease of the values of $a_{m}$ and $b_{m}$ with increasing $m$, that the system (16) and (17) may be solved by first taking $n$ equal to 1 and omitting all terms on the right in which $a$ and $b$ occur with an index greater than 1 . Thus approximate values of $a_{1}$ and $b_{1}$ are obtained. These values may then be entered in a second pair of equations obtained by taking $n=3$ and omitting all terms after those in $a_{3}$ and $b_{3}$. The approximate values of $a_{3}$ and $b_{3}$ thus obtained may be used in the first pair of equations to secure more nearly correct values of $a_{1}$ and $b_{1}$. The process may be continued indefinitely. As it appears that in general only the terms in $a_{1}$ and $b_{1}$ need be retained in (7), it is necessary to calculate only these quantities with great care. Below is given a table of the values of $a_{1}, b_{1}, a_{3}, b_{3}$, apart from the common factor $a^{3}$, calculated for various values of $\eta$ by the method just described.

\begin{tabular}{lcccr}
$\eta$ & $a_{1}$ & $b_{1}$ & $a_{3}$ & \multicolumn{1}{c}{$b_{3}$} \\
0.2 & 0.052 & 0.001 & 0.0017 & 0.00001 \\
0.35 & 0.089 & 0.006 & 0.0020 & -0.00008 \\
0.5 & 0.122 & 0.019 & 0.0015 & -0.00025 \\
0.667 & 0.148 & 0.047 & 0.0005 & -0.00055 \\
0.8 & 0.161 & 0.084 & -0.0003 & -0.00081 \\
0.9 & 0.165 & 0.121 & -0.0007 & -0.00098 \\
1.0 & 0.167 & 0.167 & -0.0010 & -0.00103
\end{tabular}

UNIVERSITY OF WISCONSIN, Madison, Wis. 